\title{
Editorial
}

\section{1 de marzo: Día Mundial del Síndrome de Down}

El 19 de diciembre de 2011 la Asamblea General de las Naciones Unidas en su $89^{\circ}$ sesión plenaria designa el 21 de marzo como el día mundial del síndrome de Down en la siguiente resolución (1):

\section{La Asamblea General,}

Recordando el Documento Final de la Cumbre Mundial 2005 y la Declaración del Milenio, así como los resultados de las grandes conferencias y cumbres de las Naciones Unidas en las esferas económica y social y otras esferas conexas,

Recordando también la Convención sobre los derechos de las personas con discapacidad, en virtud de la cual las personas con discapacidad deberán disfrutar de una vida plena y digna, en condiciones que aseguren su dignidad, fomenten su autonomía y faciliten su participación activa en la comunidad y su goce pleno de todos los derechos humanos y libertades fundamentales en igualdad de condiciones con las demás personas, y por la cual los Estados partes se comprometen a adoptar medidas inmediatas, efectivas y pertinentes para que toda la sociedad tome mayor conciencia respecto de las personas con discapacidad,

Afirmando que garantizar y promover la plena realización de todos los derechos humanos y libertades fundamentales de todas las personas con discapacidad es esencial para alcanzar los objetivos de desarrollo convenidos internacionalmente,

Consciente de que el síndrome de Down es una combinación cromosómica natural que siempre ha formado parte de la condición humana, existe en todas las regiones del mundo y habitualmente tiene efectos variables en los estilos de aprendizaje, las características físicas o la salud,

Recordando que el acceso adecuado a la atención de la salud, a los programas de intervención temprana y a la enseñanza inclusiva, así como la investigación adecuada, son vitales para el crecimiento y el desarrollo de la persona,

Reconociendo la dignidad inherente, la valía y las valiosas contribuciones de las personas con discapacidad intelectual como promotores del bienestar y de la diversidad de sus comunidades, y la importancia de su autonomía e independencia individual, en particular la libertad de tomar sus propias decisiones,

1. Decide designar el 21 de marzo Día Mundial del Síndrome de Down, que se observará todos los años a partir de 2012;

2. Invita a todos los Estados Miembros, las organizaciones competentes del sistema de las Naciones Unidas y otras organizaciones internacionales, así como a la sociedad civil, incluidas las organizaciones no gubernamentales y el sector privado, a que observen debidamente el Día Mundial del Síndrome de Down con miras a aumentar la conciencia pública sobre esta cuestión;

3. Alienta a los Estados Miembros a que adopten medidas para que toda la sociedad tome mayor conciencia, especialmente a nivel familiar, respecto de las personas con síndrome de Down;

4. Solicita al Secretario General que señale la presente resolución a la atención de todos los Estados Miembros y organizaciones de las Naciones Unidas.

En Chile se estima una prevalencia de síndrome de Down de 24,7/10.000 nacimientos, siendo la tasa más alta en Latinoamérica (2), y con una significativa tendencia al ascenso (1982-1994: 17/10.000 nacimientos vs 2001-2010: 25/10.000 nacimientos) (3). El principal factor de riesgo para esta situación epidemiológica es la postergación del embarazo a edades mayores, especialmente desde los 35 años. Es así que en nuestro país se está observando un cambio demográfico importante, caracterizado por un aumento de los nacimientos en mujeres 40 o más años (4) y un envejecimiento de las mujeres chilenas en edad fértil (5), que las expone a un mayor riesgo de malformaciones congénitas y de hijos con síndrome de Down (6).

Es conocido el mayor riesgo de mortalidad perinatal de los niños con síndrome de Down, dado por prematurez, bajo peso al nacer, óbito fetal (7) y por la asociación con comorbilidades estructurales como son las malformaciones cardíacas (8) y otras malformaciones mayores que disminuye su sobrevida a corto y mediano plazo. En la vida adulta los portadores de SD presentan otras comorbilidades 
que limitan sus expectativas de vida, especialmente insuficiencia respiratoria (9), eventos cardiovasculares mayores (10), cáncer sanguíneo y otros tumores (11).

Un estudio efectuado en países europeos entre los años 2000-2010, mostró que el 43,6\% (IC95\% $42,4-44,7 \%$ ) de los nacimientos con SD presentaban malformaciones cardíacas y el 15,0\% (IC95\% $14,2-15,8 \%$ ) presentaban otras malformaciones (12). En ese estudio hubo 14.109 casos de SD (6.738 nacidos vivos, 306 muertes fetales y 7.065 interrupciones del embarazo por anomalía fetal). De las interrupciones del embarazo solo el $8,1 \%$ (IC95\% 7,7-8,7\%) tenían una malformación cardíaca diagnosticada, y la prevalencia de las malformaciones cardíacas y otras malformaciones permanecieron constantes. Lo anterior permite concluir que la principal causa de interrupción del embarazo fue el diagnóstico prenatal de SD.

El diagnóstico prenatal de malformaciones congénitas y anomalías genéticas son un extraordinario avance en medicina materno-fetal, especialmente cuando se utiliza en mujeres de alto riesgo de presentar esas alteraciones en el desarrollo de la gestación de sus hijos ( $\geq 35$ años). El conocimiento de que su hijo/hija viene sano produce una gran tranquilidad para ella y su familia, como también es beneficioso tener el conocimiento de una anomalía estructural o genética porque permite preparar a la madre y su familia a un pronóstico perinatal adverso, como también planificar la vía de parto, y el manejo del recién nacido para un mejor diagnóstico y oportuno tratamiento.

Sin embargo, en países donde está legalizada la interrupción del embarazo, habitualmente el diagnóstico prenatal de SD termina en aborto inducido. La primera revisión sistemática sobre el tema, en 10 estudios internacionales en el período 19901998 mostró que el 92\% (IC95\% 92-93\%) de esos embarazos fueron interrumpidos (13). Una segunda revisión sistemática sobre 24 estudios realizados en EEUU, en el período 1995-2011, presentó un promedio de interrupción del embarazo de un $67 \%$ (IC95\% 61,4-93,3\%) en los estudios poblacionales y de $85 \%$ (IC95\% 60-90\%) en estudios de instituciones hospitalarias (14). En Europa (EUROCAT) la prevalencia de SD en el total de los nacimientos es de 22,0/10.000 nacimientos (IC95\% 21,7$22,4 / 10.000$ nacimientos) y de $11,2 / 10.000$ nacidos vivos (IC95\% 10,9-11,5/10.000 nacidos vivos), lo que refleja el impacto del diagnóstico prenatal en la supervivencia de los portadores de SD (15).

Pese a lo anterior, en países desarrollados, en general cuando nace una persona con SD recibe los tratamientos pertinentes a sus comorbilidades, lográndose una significativa sobrevida: en EEUU de $88,1 \%$ (IC95\% 87,0-89,0) a los 20 años (16), y en Australia de $83 \%$ (95\%IC 80-85\%) a los 30 años (17).
Es importante destacar en esos países, las políticas de inclusión de estos niños a la sociedad, es así que en España, país con una significativa reducción de personas con SD (8,09/10.000 nacidos, en 2007) se ha desarrollado el II Plan de Acción para personas con síndrome de Down en España (18), con una política de inclusión a la sociedad que establece: "Las personas con síndrome de Down tienen los mismos derechos y obligaciones que sus conciudadanos y deben disponer, al igual que éstos, de las oportunidades necesarias para su pleno desarrollo. Su diferencia debe ser aceptada y valorada positivamente para que ejerzan su derecho a desarrollar su propio proyecto de vida en un contexto caracterizado por la libre elección, la autonomía y la solidaridad". Es así que en España en mayores de 16 años, el 55,9\% es analfabeto, el $28,8 \%$ tiene estudios primarios incompletos, el $12,9 \%$ tiene estudios primarios o equivalentes y el 2,4\% estudios secundarios o superiores.

En Chile no hay estadísticas nacionales sobre prevalencia del síndrome SD, salvo cálculos estimativos proporcionados por la base de datos del $\operatorname{ECLAMC~}(2,3,6)$, como tampoco de sobrevida y de integración a la sociedad. Las encuestas de discapacidad efectuadas por el INE (19) y el Ministerio de Desarrollo Social (20) no permiten identificar al SD como causa de discapacidad. Es necesario desarrollar registros nacionales que identifiquen todas las alteraciones estructurales (malformaciones congénitas) y anomalías cromosómicas (trisomías $21,13,18$ y otras) diagnosticadas durante la gestación y al nacer para tener una real dimensión de la incidencia y prevalencia de estas condiciones, con fines de que se puedan desarrollar programas y políticas públicas del tratamiento de sus comorbilidades y de inclusión a la sociedad.

La dignidad de las personas con SD no empieza al momento de nacer sino desde la concepción.

Dr. Enrique Donoso S. Editor Jefe.

Revista Chilena de Obstetricia y Ginecología.

\section{REFERENCIAS}

1. ONU. Resolución aprobada por la Asamblea General el 19 de diciembre de 2011. A/RES/66/149. Día Mundial del Síndrome de Down. Disponible en: http:// www.un.org/es/comun/docs/?symbol=A/RES/66/149.

2. Nazer J, Cifuentes L. Malformaciones congénitas en Chile y Latino América: Una visión epidemiológica del ECLAMC del período 1995-2008. Rev Méd Chile 2011;139:72-8.

3. Nazer J, Cifuentes L. Estudio epidemiológico global del síndrome de Down. Rev Chil Pediatr 2011;82:10512.

4. Donoso E, Becker J, Villarroel L. Evolución de la natalidad y del riesgo reproductivo en mujeres de 40 o 
más años en la década de los 90 . Rev Chil Obstet Ginecol 2002;67(2):139-42.

5. Donoso E, Carvajal J, Domínguez A. Reducción de la fecundidad y envejecimiento de la población de mujeres chilenas en edad fértil: 1990-2004. Rev Méd Chile 2009;137:766-73.

6. Nazer J, Cifuentes L, Águila A, Ureta P, Bello MP, Correa F, Melibosky F. Edad materna y malformaciones congénitas. Un registro de 35 años. 1970-2005. Rev Méd Chile 2007;135:1463-9.

7. Sparks TN, Griffin E, Page J, Pilliod R, Shaffer BL, Caughey $A B$. Down syndrome: perinatal mortality risks with each additional week of expectant management. Prenat Diagn 2016. doi: 10.1002/pd.4792.

8. Lizama Calvo M, Cerda Lorca J, Monge Iriarte M, Carrillo Mayanquer I, Clavería Rodríguez C, Castillo Moya A. [Hospital morbidity and mortality in children with Down's syndrome: Experience in a university hospital in Chile]. Rev Chil Pediatr 2015. pii: S03704106(15)00261-2. doi: 10.1016/j.rchipe.2015.06.026.

9. Huppal H, Chandran S, Potluri R. Risk factor for mortality in Down syndrome. JIDR 2015;59:873-81.

10. Sobey GCh, Judkins CP, Sundararajan V, Phan TG, Drummond GR, Srikanth VK. Risk of major cardiovascular events in people with Down syndrome. PLoS One 2015;10(9)1-10.

11. Hill DA, Gridley G, Cnattingius S, Mellemkjaer L, Linet $\mathrm{M}$, Adami $\mathrm{H}-\mathrm{O}$, et al. Mortality and cancer incidence among individuals with Down syndrome. Arch Intern Med 2003;163:705-11.

12. Morris JK, Garne E, Wellesley D, Addor MC, Arriola L, Barisic I, et al. Major congenital anomalies in babies born with Down syndrome: a EUROCAT population-based registry study. Am J Med Genet A 2014;164A(12):2979-86.

13. Mansfield C, Hopfer S, Marteau TM. Termination rates after prenatal diagnosis of Down syndrome, spina bifida, anencephaly, and Turner and Klinefelter syndromes: a systematic literature review. European Concerted Action: DADA (Decision-making After the Diagnosis of a fetal Abnormality). Prenat Diagn 1999;19(9):808-12.

14. Natoli JL, Ackerman DL, McDermott S, Edwards JG. Prenatal diagnosis of Down syndrome: a systematic review of termination rates (1995-2011). Prenatal Diagnosis 2012;32(2):142-53.

15. Loane M, Joan K Morris JK, Addor MC, Arriola L, et al. Twenty-year trends in the prevalence of Down syndrome and other trisomies in Europe: impact of maternal age and prenatal screening. Eur J Hum Genet 2013; 21(1):27-33.

16. Kucik JE, Shin M, Siffel C, Marengo L, Correa A; Congenital Anomaly Multistate Prevalence and Survival Collaborative. Trends in survival among children with Down syndrome in 10 regions of the United States. Pediatrics 2013;131(1):e27-36.

17. Glasson EJ, Jacques A, Wong K, Bourke J, Leonard $\mathrm{H}$. Improved Survival in Down Syndrome over the Last 60 Years and the Impact of Perinatal Factors in Recent Decades. J Pediatr 2016;169:214-220.e1.

18. Federación Española de Síndrome de Down. II Plan de Acción para personas con síndrome de Down en España (2009-2013). Disponible en: http://www.sindromedown.net/wp-content/uploads/2014/09/56L_iiplande.pdf

19. Instituto Nacional de Estadísticas (INE). Primer Estudio Nacional de la discapacidad e informes regionales 2004. Disponible en: http://www.ine.cl/canales/chile_ estadistico/encuestas_discapacidad/discapacidad. php.

20. Ministerio de Desarrollo Social. Gobierno de Chile. CASEN 2013. Inclusión social de personas con dificultades y/o condiciones permanentes y de larga duración (26 de abril 2015). Disponible en: http://observatorio.ministeriodesarrollosocial.gob.cl/documentos/ Casen2013_inclusion_social.pdf. 